\title{
Trichostatin A inhibits proliferation of triple negative breast cancer cells by inducing cell cycle arrest and apoptosis
}

\author{
X. SONG ${ }^{1}$, J. Q. WU ${ }^{1}$, X. F. YU ${ }^{1,2, *}$, X. S. YANG ${ }^{3, *}$, Y. YANG ${ }^{1, *}$ \\ ${ }^{1}$ Department of Microbiology and Immunology, School of Life Sciences, Tianjin University, Tianjin, China; ${ }^{2}$ Cancer Institute (Key Laboratory \\ of Cancer Prevention and Intervention, China National Ministry of Education), The Second Affiliated Hospital, Zhejiang University School of \\ Medicine, Hangzhou, Zhejiang, China; ${ }^{3}$ Department of Radiation Prevention, School of Public Health, Jilin University, Changchun, Jilin, China
}

*Correspondence: yangy@tju.edu.cn,13844872707@163.com,xyu2@jhu.edu

Received July 31, 2017 / Accepted April 11, 2018

\begin{abstract}
Triple negative breast cancer (TNBC) is the most aggressive subtype of breast cancer with poor outcome. Because of lacking therapeutic targets, chemotherapy is the main treatment option for patients with TNBC. Overexpression of HDACs correlates with tumorigenesis, highlighting the potential of HDACs as therapeutic targets for TNBC. Here we demonstrate that trichostatin A (TSA, a HDAC inhibitor) selectively inhibits the proliferation of TNBC cell lines HCC1806 and HCC38 rather than a normal breast cell line MCF10A. The inhibition of TNBC by TSA is via its roles in inducing cell cycle arrest and apoptosis. TSA treatment leads to decreased expression of CYCLIN D1, CDK4, CDK6 and BCL-XL, but increased P21 expression. Moreover, combination of TSA with doxorubicin has synergistic effects on inhibiting proliferation of HCC1806 and HCC38 cells. Our studies identified a promising epigenetic-based therapeutic strategy that may be implemented in the therapy of fatal human breast cancer.
\end{abstract}

Key words: triple negative breast cancer, Trichostatin A, apoptosis; cell cycle arrest, MAPK

Triple negative breast cancer (TNBC) is one of the most aggressive human cancers associated with early metastasis and death [1-3]. Because TNBC do not express estrogen receptor (ER), progesterone receptor (PR), and human epidermal growth factor 2 (HER2), patients with TNBC cannot benefit from the current anti-estrogen and anti-HER2 antibody therapies $[4,5]$. Currently, the standard of care is surgery with adjuvant chemotherapy or radiation therapy. Therefore, new therapeutic strategy of TNBC is urgently needed to decrease the side effect and resistance of chemotherapy.

TNBC is a heterogeneous disease and categorized into five major subtypes based on gene expression profile: basal like, immunomodulatory, mesenchymal, mesenchymal stem like and luminal androgen receptor [6]. Basal like TNBC accounting for $47 \%$, is the main type of TNBC. The top gene ontologies for the basal like breast cancer subtype are heavily enriched in cell cycle and cell division components and pathways [7]. Therefore, targeting cell cycle related genes might be effective approach to treat basal like breast cancers.

Epigenetic mechanisms such as DNA methylation and histone modification play important roles in tumorigen- esis. The histone deacetylase (HDAC) remodel chromatin and regulate genes transcription by removing acetyl groups from histone and further modulate the process of cell proliferation, cell cycle and cell differentiation $[8,9]$. Multiple tumors express higher level of HDACs which indicate that HDACs might be potential targets for epigenetic treatment [10]. HDAC inhibitors enhance the acetylation of cellular proteins by blocking HDAC activity and play critical roles against cancer by inducing differentiation, cell cycle arrest and apoptosis $[11,12]$. Trichostatin A (TSA) is a pan-HDAC inhibitor and exert anti-tumor effect in multiple cancers, such as colon carcinoma cells, breast adenocarcinoma cells, prostate cancer cells and esophageal squamous cells [13-18]. Yang et al demonstrated that TSA enhance sensitivity of ERa-negative breast cancer to chemotherapy by inducing the expression of ERa [19]. The mechanisms of TSA on TNBC are still under investigation.

In this study, we demonstrate that TSA inhibit proliferation of basal like breast cancer cells through inducing cell cycle arrest and apoptosis. The expressions of CYCLIN D1, CDK4, CDK6 and BCL-XL were down-regulated, while the expression of $\mathrm{P} 21$ protein was enhanced. Combination of TSA and doxorubicin showed a synergistically effects on 
inhibiting the proliferation of basal like breast cancer cells. Our study will benefit for TNBC therapy in the future.

\section{Materials and methods}

Chemicals and antibodies. TSA were purchased from Sigma-Aldrich. Stock solution of TSA was prepared with DMSO. Final concentration of DMSO in assay media was typically kept at $0.1 \%$. Doxorubicin was purchased from Selleck. Antibodies against CDK4, CDK6, CYCLIN D1, P21, p-ERK, ERK, p-AKT, AKT, cleaved caspase3, PARP and $\beta$-Actin were all purchased from Cell Signaling Technology.

Cell culture. Basal like TNBC cell lines HCC38 and HCC1806 were cultured in RPMI 1640 supplemented with $10 \%$ FBS. The cell lines were maintained at $37^{\circ} \mathrm{C}$ in a humidified atmosphere with 5\% CO2. Normal mammary epithelial cell line MCF-10A cells were cultured in DMEM/F12 supplemented with $20 \mathrm{ng} / \mathrm{ml}$ of epidermal growth factor, $100 \mathrm{ng} / \mathrm{ml}$ of cholera toxin, $0.01 \mathrm{mg} / \mathrm{ml}$ of insulin, $500 \mathrm{ng} / \mathrm{ml}$ of hydrocortisone, and $5 \%$ horse serum.

MTT assay. MTT [3-(4,5-Dimethylthiazol-2-yl)-2,5-diphenyl tetrazolium] dye reduction method was conducted to detect the cell viability as described previously [20]. Briefly, 3000 cells in the log phase of growth were plated into 96-well plates. Cells were exposed to the indicated doses of TSA for $72 \mathrm{~h}$. Time-dependent effects on cells were tested at $24 \mathrm{~h}, 48 \mathrm{~h}$ and $72 \mathrm{~h}$ after treatment. The absorbance value (OD) of the wells was measured with a microplate reader with wavelength of $570 \mathrm{~nm}$. Triplicate samples was performed each test.

Cell cycle assay. Cells $\left(8 \times 10^{5}\right)$ were plated in $6 \mathrm{~cm}$ dishes and treated with indicated dose of TSA or vehicle for $24 \mathrm{~h}$. Then cells were harvested and fixed in $70 \%$ of cold ethanol overnight. Cells were washed with PBS three times and incubated with RNase at $37^{\circ} \mathrm{C}$ for 30 minutes followed by Propidium Iodide (PI) staining for another 30 minutes at $4{ }^{\circ} \mathrm{C}$. Cell suspensions were analyzed by flow cytometry. Data were analyzed with Modifit software.

Immunoblotting analysis. Cells $\left(8 \times 10^{5}\right)$ were plated in $6 \mathrm{~cm}$ dishes and treated with indicated dose of TSA or vehicle for $24 \mathrm{~h}$. Cells were washed with cold PBS and lysed in cell lysis buffer (50 mM Tris, pH7.4, with $150 \mathrm{mM} \mathrm{NaCl}, 1 \%$ NP40, $9 \mathrm{mM}$ ethylenediamine tetra-acetic acid [EDTA], and protease inhibitor cocktail [Roche]), spin down at $12000 \mathrm{rpm}$ to collect the protein supernatant. The protein concentration was analyzed by a protein assay kit with bovine serum albumin standards according to the manufacturer's instructions (Bio-Rad Laboratories, Hurcules, CA). Cell lysate was separated by SDS-PAGE and transferred onto a nitrocellulose membrane (Hybond-C, Amersham Pharmacia Biotech, Inc., Piscataway, NJ). Following blocking with PBS-Tween-20 containing 5\% nonfat dry milk for 1 hour, membranes were incubated overnight at $4{ }^{\circ} \mathrm{C}$ with primary antibodies followed by incubation with horseradish peroxidase-conjugated secondary antibody. $\beta$-actin was used as loading control. Immunoreactive bands were detected by an enhanced chemiluminescence kit. Grayscale analysis was done by ImageJ software. The control group was set as 1 . The ratio of grayscale was calculated vs. control.

Statistical analysis. Statistical analyses were conducted using SPSS 22.0 software package (SPSS Inc., Chicago, IL, USA). Data from experiments were presented as means $\pm S D$, and evaluated by analysis on factorial design of two factors and one-way ANOVA. Differences were considered statistically significant at $\mathrm{p}<0.05$.

\section{Results}

TSA inhibited proliferation of basal like TNBC cell lines HCC1806 and HCC38. Side effect is the main hurdle of chemotherapy for clinical use. First of all, it is important to demonstrate that TSA inhibit the proliferation of breast cancer cells with less toxicity on normal breast cells. Here MCF10A, a normal mammary epithelial cell line, was used to detect the toxicity of TSA on normal breast cells. TSA showed a 5\% 25\% inhibitions on MCF10A cell viability while the inhibition rate of $0.5 \mu \mathrm{M}$ of TSA on HCC1806 and HCC38 breast cells is 95\% (Figures 1A-1C). Moreover, TSA inhibit HCC1806 and HCC38 cells in dose-dependent and time-dependent patterns (Figures $1 \mathrm{D}$ and $1 \mathrm{E}$ ). It indicates the potential of TSA on TNBC therapy in the near future.

TSA induced cell cycle arrest by down-regulation of CDK4, CDK6 and CYCLIND1 expression and up-regulation of P21 expression. Next, we demonstrate whether TSA inhibit the proliferation of breast cancer cells through inducing cell cycle arrest. $0.13 \mu \mathrm{M}$ of TSA was used for detecting the cell cycle arrest in TNBC cells. The results showed that both G0/G1 and G2/M arrest were observed in HCC1806 cell line at 24 hours after TSA treatment (Figures 2A and 2B) compared with DMSO control $(\mathrm{p}<0.01$ vs. control). While only G0/G1 arrest was observed in HCC38 cells (Figures $3 \mathrm{~A}$ and $3 \mathrm{~B}$ ).

In order to explore the molecular basis of cell cycle arrest induced by TSA, we further examined CDK4, CDK6, CYCLIN D1 and P21 protein expression, which are the key regulators participating in G1 to S phase transition. Our data showed that TSA reduced the expression of CDK4, CDK6 and CYCLIN D1, while cyclin-dependent kinase inhibitor, P21 protein, was increased in both HCC1806 and HCC38 cell lines (Figure 2C and Figure 3C).

TSA induced apoptosis by down-regulation of BCL-XL expression. In addition to block cell cycle transition, it has been previously demonstrated that TSA induced cell apoptosis in some tumor cell lines. We further identify whether TSA induces cell apoptosis in HCC1806 and HCC38 cells. Both cells were treated with TSA for 24 hours and western blot was used to check the expression of apoptosis-related proteins. TSA induces cleaved CASPASE3 and cleaved PARP expression in both cells in a dose dependent manner (Figures $4 \mathrm{~A}$ and $4 \mathrm{~B}$ ). Cleaved CASPASE3 was increased about 35 fold and 12.5 fold in HCC1806 cells and HCC38 cells treated by 
A

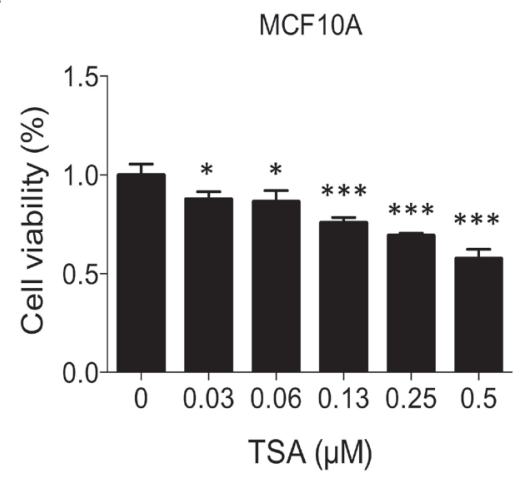

C

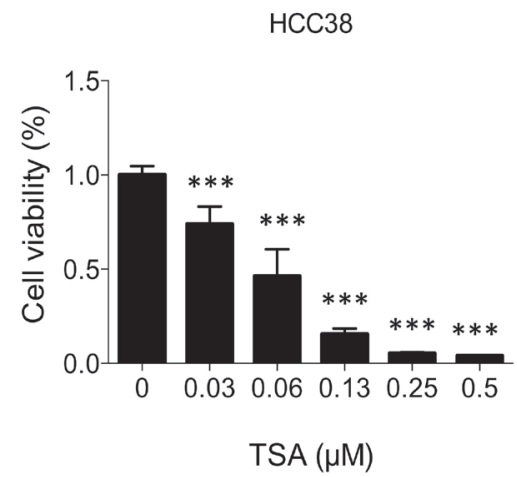

B

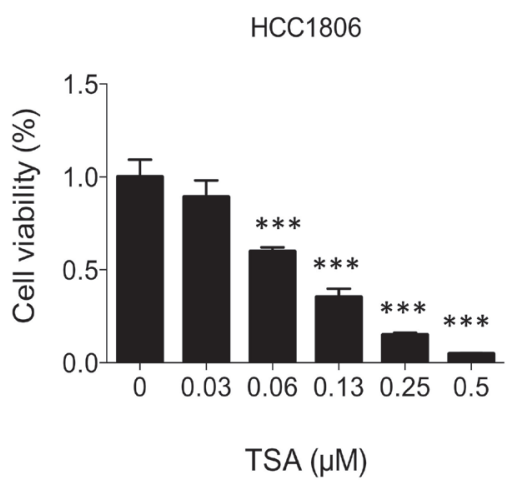

D

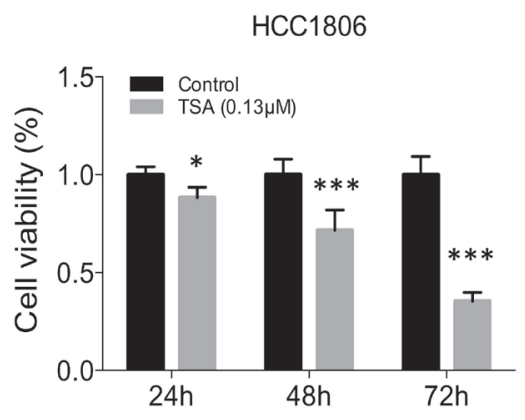

E

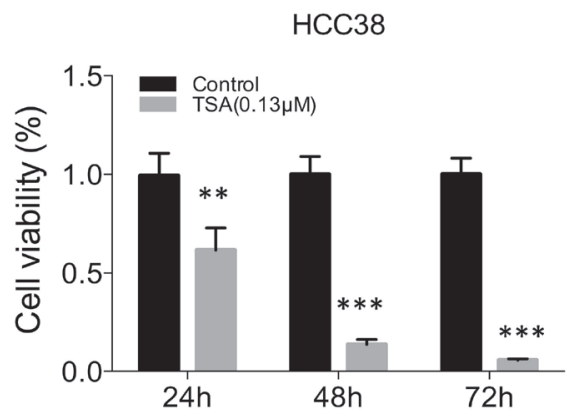

Figure 1. The inhibitory effects of TSA on MCF10A, HCC1806 and HCC38 cells. A-C. MCF10A cells, HCC1806 and HCC38 cells were treated with TSA for $72 \mathrm{~h}$ and MTT assay was used to detect the cell viability. D-E. Time-dependent effects of TSA on HCC1806 and HCC38 cells. HCC1806 and HCC38 cells were treated with $0.13 \mu \mathrm{M}$ of TSA for $24 \mathrm{~h}, 48 \mathrm{~h}$ and $72 \mathrm{~h}$ respectively. MTT assay was used to detect the cell viability.

Note: $\mathrm{n}=3,{ }^{*} \mathrm{p}<0.05,{ }^{* *} \mathrm{p}<0.01,{ }^{* * *} \mathrm{p}<\mathbf{0 . 0 0 5}$ significantly different compared with control.

$0.5 \mu \mathrm{M}$ of TSA respectively. $0.25 \mu \mathrm{M}$ of TSA cause more than ten-fold increase of cleaved PARP compared with control in both cell lines (11.02 fold in HCC1806 cells, 10.49 fold in HCC38 cells). Furthermore, we investigated the underlying mechanism of cell apoptosis. The expression of BAX and BCL-XL protein were detected in both HCC1806 and HCC38 cells followed by TSA treatment. Down-regulation of BCL-XL protein was induced in HCC1806 cells by TSA treatment, while no change of BAX protein was observed. But the BCL-XL was down-regulated and BAX protein was increased in HCC38 cells (Figure 4). These results indicate that apoptosis were induced by TSA treatment in TNBC through distinct mechanisms.

TSA disrupted MAPK pathways. PI3K and MAPK pathways play important roles in cell proliferation and survival. To investigate the underlying mechanism of TSA on basal like breast cancer cells, we detect the expression of p-ERK, ERK, p-AKT and AKT protein in HCC1806 and HCC38 cells treated with TSA. TSA decreased phosphorylation of ERK in both cells (Figures 5A and 5B). The expression of pAKT was decreased only at higher dose of TSA $(0.5 \mu \mathrm{M})$ treatment. It indicated that down-regulation of MAPK cell 
A

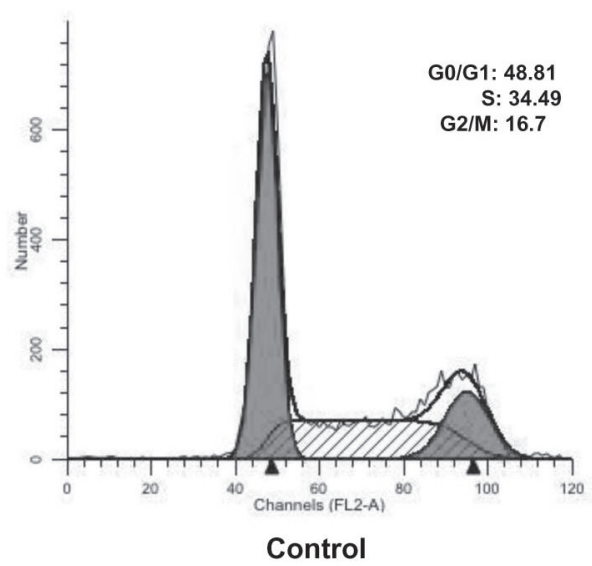

B

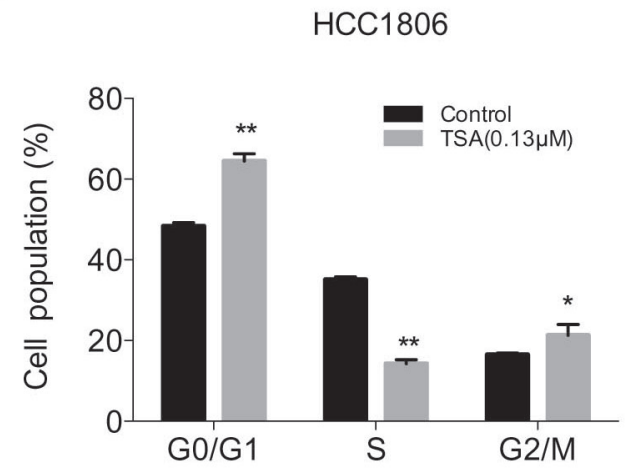

HCC1806

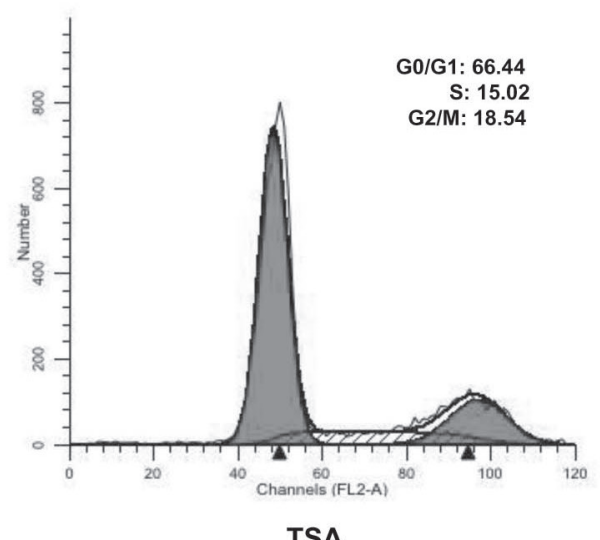

C

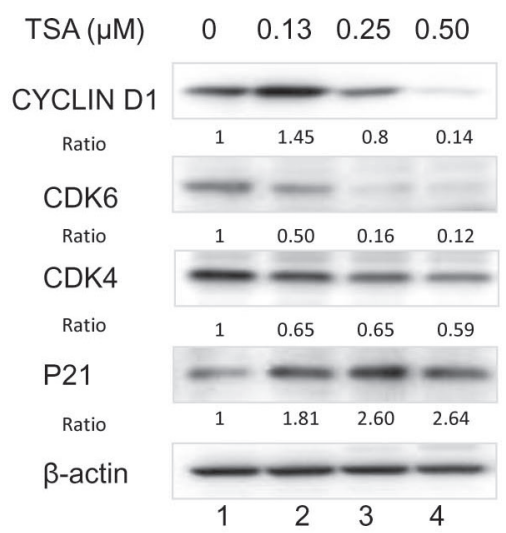

Figure 2. TSA induced cell cycle arrest in HCC1806 cells. A-B. HCC1806 cells were treated with TSA $(0.13 \mu \mathrm{M})$ and flow cytometry was used to detect the cell cycle of HCC1806 cells 24 hours after treatment. Modifit software was used to analyze the data. G0/G1 arrest was observed in HCC1806 cells treated with TSA, $p<0.01$, vs. control. C. The effects of TSA on cell cycle proteins in HCC1806 cells. HCC1806 cells were treated with TSA and cells were collected at $24 \mathrm{~h}$ after treatment and western blot was conducted to detect the expression of proteins. The grayscale value was labeled below the band. Note: $n=3,{ }^{*} p<0.05,{ }^{* *} p<0.01$ significantly different compared with control.

signaling pathway attributed to the suppression of cell proliferation and survival and PI3K cell signaling pathway is not significantly affected by TSA.

TSA and doxorubicin enhanced apoptosis synergistically in HCC1806 cells and HCC38 cells. Doxorubicin (Dox), an anthracycline antibiotic, was routinely used in treating breast cancer patients in clinic. However, it has been limited by the risk of cardiotoxicity and other lethal side effects. Combination therapy is a widely used strategy to increase efficacy and decrease side effects of chemotherapy. Therefore, we assessed the synergistic effect of TSA and doxorubicin on HCC1806 and HCC38 cells proliferation. Herein, we used $0.03 \mu \mathrm{M}$ of TSA combined with different doses of doxorubicin. Notably, combination of doxorubicin and TSA showed more potential inhibitory effects than either compound alone on proliferation of HCC1806 and HCC38 cells (Figure 6A). The expression of cleaved PARP protein and cleaved CASPASE3 protein were higher than those of treatment with TSA or doxorubicin alone in HCC1806 cells (Figure 6B). Moreover, the expression of pERK protein is reduced significantly while pAKT has no change (Figure 6C). It indicated more effective in combination group than the single group alone.

\section{Discussion}

Here we demonstrated that HDAC small-molecule inhibitor TSA and doxorubicin have a potent synergistic tumorsuppressive effect on human basal like TNBC cell lines. Therapies commonly used in other breast cancer subtypes are ineffective for TNBC. The improvement of efficacy of current chemotherapy for TNBC is urgently needed [20]. Recent studies focus on incorporation of platinum salts and new combinations of conventional chemotherapeutic agents with 
A

HCC38

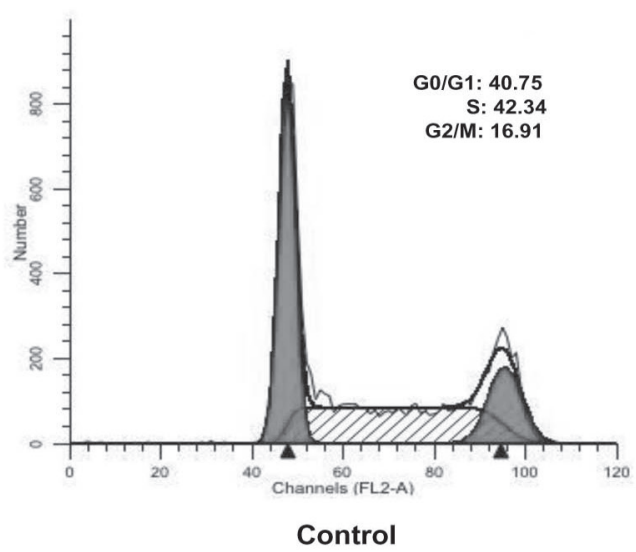

B

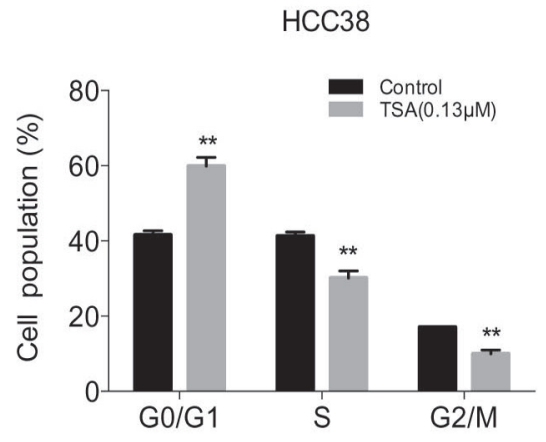

HCC38

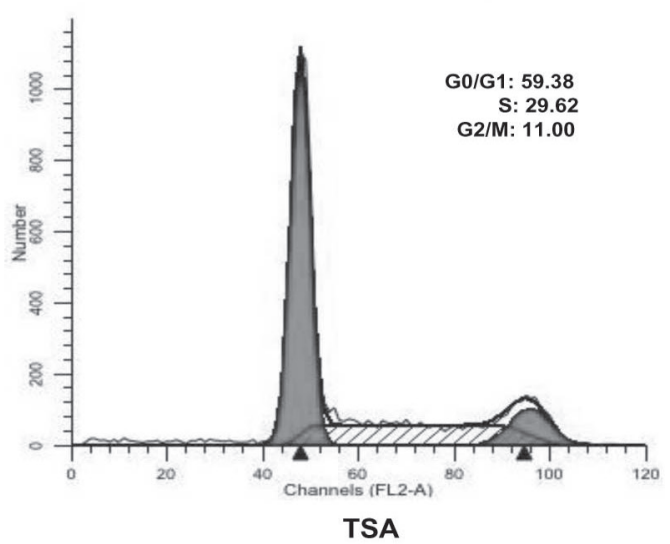

C

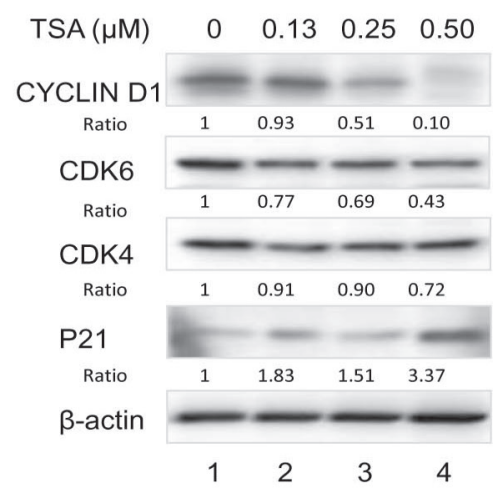

Figure 3. TSA induced cell cycle arrest in HCC38 cells. A-B. HCC38 cells were treated with TSA $(0.13 \mu \mathrm{M})$ and flow cytometry was used to detect the cell cycle of $\mathrm{HCC} 38$ cells 24 hours after treatment. Modifit software was used to analyze the data. G0/G1 arrest was observed in HCC38 cells treated with TSA, $p<0.01$, vs. control. C. The effects of TSA on cell cycle proteins in HCC38 cells. HCC38 cells were treated with TSA as the same as HCC1806 cells. The grayscale value was labeled below the band. Note: $n=3,{ }^{*} p<0.05,{ }^{* *} p<0.01$ significantly different compared with control.

new-targeted agents, such as poly-ADP ribose polymerase inhibitors, antiangiogenic agents, phosphoinositide 3-kinase (PI3K) pathway inhibitors, and androgen antagonist [21].

TSA, a HDAC inhibitor, exerted a potent activity against human colon carcinoma cells, breast adenocarcinoma cells and prostate cancer cells through inducing cell cycle arrest, differentiation and apoptosis [16, 18, 22]. Mitotic cellular division requires the cell to leave the resting state and proceed through phases of DNA synthesis and mitosis. Wellorganized progression of dividing cells through the G1, S, $\mathrm{G} 2$, and M phases of the cell cycle in eukaryotic cells relies on a series of cell-cycle regulatory proteins, such as CYCLIN A, B, D and E. Cyclins exert their functions via activating a variety of specific cyclin-dependent kinases (CDKs) [5]. The cell cycle in mammals is controlled by interaction of cyclins and cyclin dependent kinases (CDKs), and CDK inhibitors such as P21 and P27. CDK inhibitors inhibited the activity of
cyclin-CDK complexes and blocked cell cycle transition [23]. CYCLIN D1 is a key regulator of G1/S checkpoint control, which forms a holoenzyme complex with CDK4 and CDK6, to phosphorylate $\mathrm{pRB}$ (retinoblastoma protein). When RB was phosphorylated, transcription factor E2F was released from pRB to promote cell cycle progress $[24,25]$. In this study, we observed that TSA treatment resulted in downregulation of CYCLIN D1, CDK4 and CDK6 protein expression, but up-regulation of $\mathrm{P} 21$ protein expression, which is the underlying mechanism of the subsequent growth inhibition effect of TSA.

In addition, HDAC inhibitors induce apoptosis through mitochondrial-mediated apoptosis pathway $[16,17,26]$. In the mitochondrial pathway, the ratio of BAX to BCL2 or BCL-XL was disrupted in mitochondrial membrane, promoting the release of cytochrome $\mathrm{C}$ and other proapoptotic molecules into the cytoplasm, which in turn lead 
A

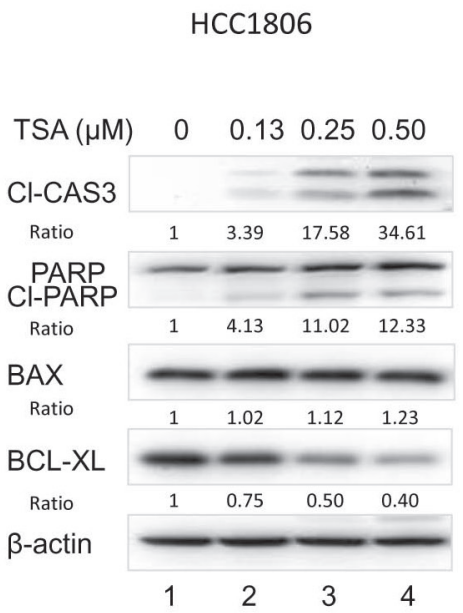

B

HCC38

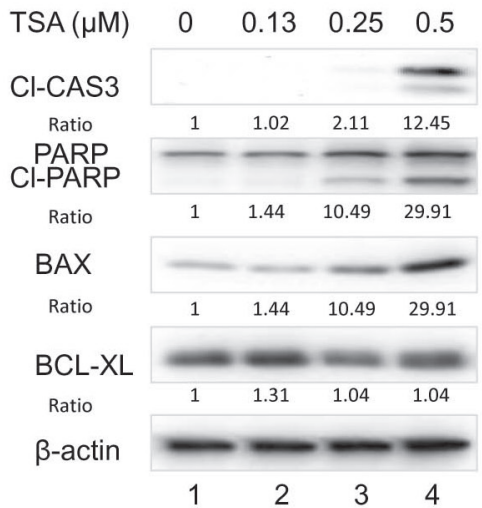

Figure 4. TSA induced apoptosis in HCC1806 and HCC38 cells. HCC1806 (A) and HCC38 (B) cells were treated with TSA and cells were collected 24 $\mathrm{h}$ after treatment. Cleaved-CASPASE3 (Cl-CAS3), cleaved-PARP(Cl-PARP), BAX and BCL-XL were detected. The grayscale value was labeled below the band.

A

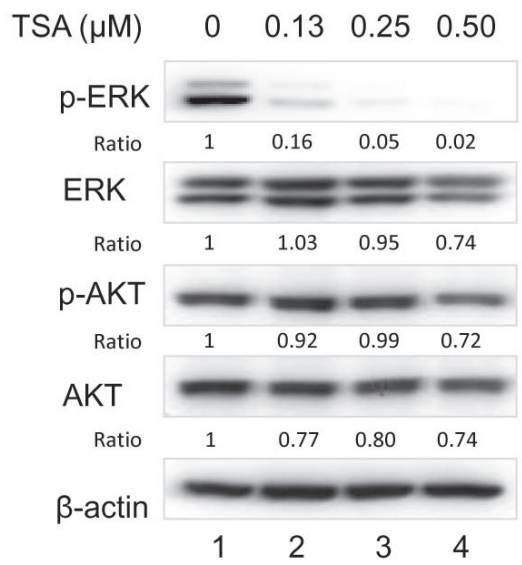

B

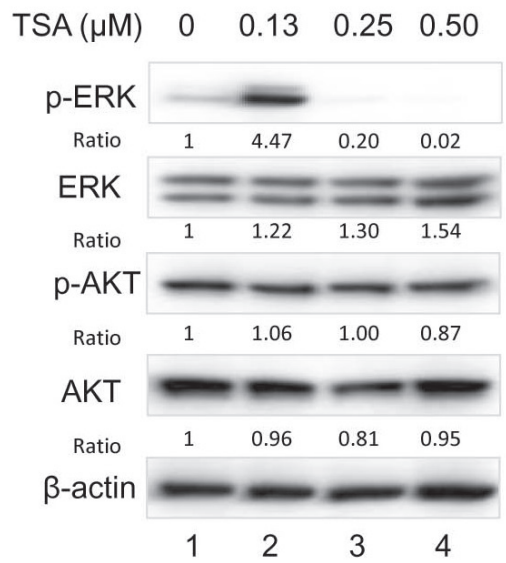

Figure 5. TSA inhibited cells growth by disrupting MAPK pathway. HCC1806 (A) and HCC38 (B) cells were treated with TSA for $24 \mathrm{~h}$ and cells were lysed with lysis buffer for evaluating the expression of the cell signaling proteins by western blot. The expression of phosphorylated ERK (pERK) represents the activation of MAPK pathway. Phosphorylated AKT (pAKT) is the major regulator of PI3K pathway. The grayscale value was labeled below the band.

to the activation of CASPASE 9 to activate CASPASE 3 and further to cleave PARP. Herein, we demonstrated that TSA treatment promoted the expression of cleaved CASPASE 3 and cleaved PARP, which indicated TSA inducing the cell apoptosis. Furthermore, we demonstrated TSA decreased the expression of anti-apoptotic protein BCL-XL. It suggested that TSA mediated apoptosis through activating the mitochondrial-mediated apoptosis pathway.
It is well known that PI3K and MAPK signaling pathways are closely related to cell proliferation, differentiation and survival [27, 28]. It was reported that HDAC inhibitor decreased phosphorylation of AKT in diffuse large B-cell lymphoma cell lines [5]. We found that TSA decreased the expression of phosphorylation of ERK, but not phosphorylation of AKT in basal like breast cancer cell line. These results indicated that suppressing the MAPK signaling pathways 
in basal like breast cancer is responsible for the cell growth inhibition induced by TSA. Crucial preclinical trials on HDAC inhibitors (panobinostat, vorinostat, and entinostat) exert an anti-proliferative effect on TNBC cells and control tumor growth by multiple mechanisms of action, including apoptosis and regulation of the epithelial to mesenchymal transition (EMT). HDAC inhibitors such as suberoylanilidehydroxamic acid (SAHA), sodium butyrate, mocetinostat, panobinostat, entinostat, YCW1 and N-(2-hydroxyphenyl)2-propylpentanamide have shown promising therapeutic outcomes against TNBC, especially when they are used in combination with other anticancer agents $[29,30]$.

Drug combination is a widely used strategy to increase efficacy and decrease side effects of chemotherapy [31]. Doxorubicin is widely used in cancer therapy in clinic. Herein we explored synergistic inhibitory effect of TSA and Doxorubicin on TNBC cell lines. The combination of TSA and doxorubicin exerts synergistic effects in reducing basal like breast cancer cells viability by inducing cleavage of PARP and CASPASE 3 that is associated with cell apoptosis.

In general, HDACs can be divided into $\mathrm{Zn}^{2+}$ - dependent classes (class I, II and IV) and NAD-dependent classes (class III). Class I is consisted of HDACs 1, 2, 3 and 8. Class II can be divided further into class IIa (HDAC4, 5, 7, and 9) and class IIb (HDAC6 and 10). Class III members consist of SIRT1SIRT7. HDAC11 is the only member of Class IV. In this study, we conduct pan- HDAC inhibitor, TSA, and explored the possible inhibitory mechanisms of TSA on TNBC. TSA can effectively inhibit TNBC proliferation and it indicates that TSA inhibit TNBC through regulating the epigenetic process (including histone acetylation and deacetylation) and receptor-independent pathway (including ER, PR and HER2 receptors). Hsieh, et al found HDAC3 linked to CSC homeostasis by increasing $\beta$-catenin expression through the Akt/GSK3 $\beta$ pathway [32]. Witt et al demonstrate that clinically available HDAC inhibitors (HDACi) targeting HDAC1 and HDAC7 can be used to preferentially target CSCs [33]. Although we observed promising effects of TSA combined with doxorubicin, it is necessary to search for the specific HDAC target and develop the target specific inhibitors.

In conclusion, HDAC inhibitor TSA had an inhibitory effect on basal like breast cancer cell line by inducing cell cycle arrest and apoptosis and inhibiting the MAPK pathway. TSA and doxorubicin combination exerts synergistic effects in reducing basal like breast cancer cells viability. Our study supports the rationale for TSA alone or in combination as

A

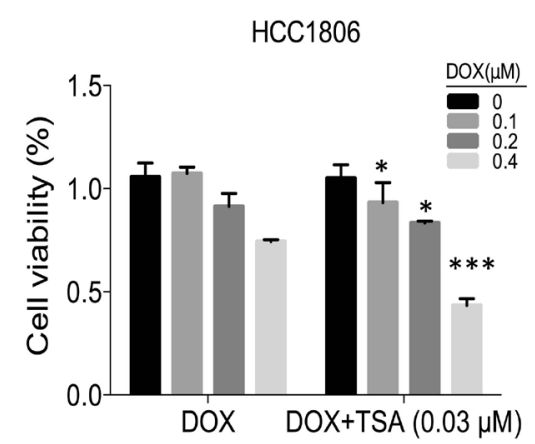

B

C
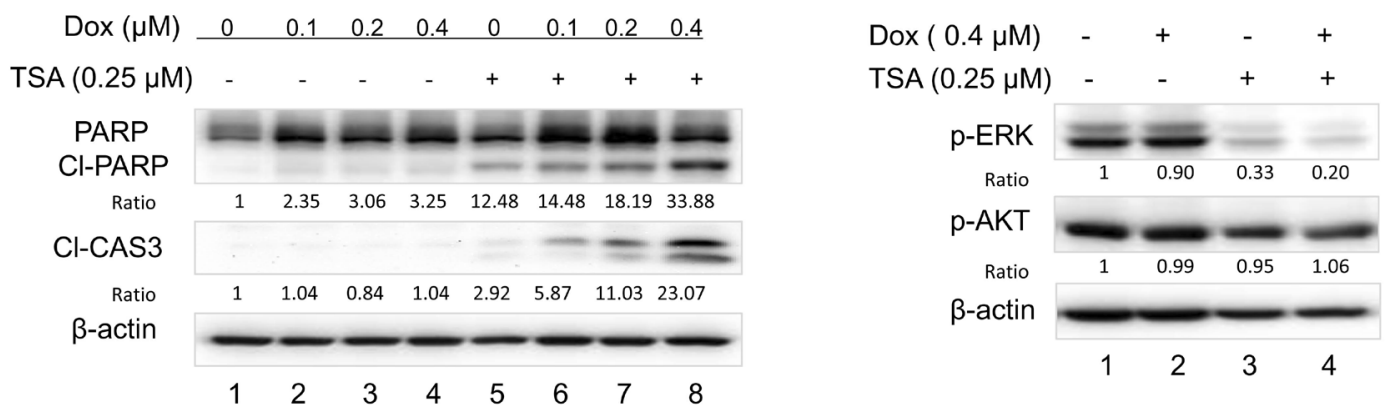

Figure 6. Synergistic effects of TSA with Doxorubicin. A. The effects of TSA combined with doxorubicin on cell viability of HCC1806 cells. HCC1806 cells were treated with doxorubicin $(0 \mu \mathrm{M}, 0.1 \mu \mathrm{M}, 0.2 \mu \mathrm{M}$ and $0.4 \mu \mathrm{M})$ and TSA $(0.03 \mu \mathrm{M})$ for $72 \mathrm{~h}$ and MTT assay was used to detect the cell viability. Statistical analysis was done between the group of doxorubicin combined with TSA and the same dose of doxorubicin treatment only group. $\mathbf{n}=3$, ${ }^{*} \mathrm{p}<0.05,{ }^{* *} \mathrm{p}<0.01,{ }^{* *} \mathrm{p}<0.005$. B. The effects of TSA combined with doxorubicin on apoptosis in HCC1806 cells. HCC1806 cells were treated with $0.25 \mu \mathrm{M}$ of TSA and doxorubicin $(0 \mu \mathrm{M}, 0.1 \mu \mathrm{M}, 0.2 \mu \mathrm{M}$ and $0.4 \mu \mathrm{M})$. The grayscale calculation was done as the same as above. C. The effects of TSA on MAPK and PI3K cell signaling pathway. HCC1806 cells were treated with $0.25 \mu \mathrm{M}$ of TSA and $0.4 \mu \mathrm{M}$ of doxorubicin for 24 h. Cells were lysed with lysis buffer and western blot was conducted to detect the protein expression. The grayscale value was labeled below the band. 
a promising therapeutic approach against basal like breast cancer which are largely refractory to current therapeutic approaches, and will hopefully promote the rapid clinical evaluation of the strategy.

Acknowledgements: This study is supported by Tianjin Applied Basic and Advanced Technology Research Program (Project Approval Number: 13JCYBJC40800).

\section{References}

[1] DENT R, TRUDEAU M, PRITCHARD KI, HANNA WM, KAHN HK et al. Triple-negative breast cancer: clinical features and patterns of recurrence. Clin Cancer Res 2007; 13: 4429-4434. https://doi.org/10.1158/1078-0432.CCR-063045

[2] OAKMAN C, VIALE G, Di LEO A. Management of triple negative breast cancer. Breast 2010; 19: 312-321. https://doi. org/10.1016/j.breast.2010.03.026

[3] BADVE S, DABBS DJ, SCHNITT SJ, BAEHNER FL, DECKER T et al. Basal-like and triple-negative breast cancers: a critical review with an emphasis on the implications for pathologists and oncologists. Mod Pathol 2011; 24: 157-167. https://doi.org/10.1038/modpathol.2010.200

[4] YADAV BS, SHARMA SC, CHANANA P, JHAMB S. Systemic treatment strategies for triple-negative breast cancer. World J Clin Oncol 2014; 5: 125-133. https://doi. org/10.5306/wjco.v5.i2.125

[5] CAI Y, CUI W, CHEN W, WEI P, CHI Y et al. The effects of a histone deacetylase inhibitor on biological behavior of diffuse large B-cell lymphoma cell lines and insights into the underlying mechanisms. Cancer Cell Int 2013; 13: 57. https://doi.org/10.1186/1475-2867-13-57

[6] LEHMANN BD, BAUER JA, CHEN X, SANDERS ME, CHAKRAVARTHY AB et al. Identification of human triplenegative breast cancer subtypes and preclinical models for selection of targeted therapies. J Clin Invest 2011; 121: 27502767. https://doi.org/10.1172/JCI45014

[7] AHN SG, KIM SJ, KIM C, JEONG J. Molecular Classification of Triple-Negative Breast Cancer. J Breast Cancer 2016; 19: 223-230. https://doi.org/10.4048/jbc.2016.19.3.223

[8] MINUCCI S, PELICCI PG. Histone deacetylase inhibitors and the promise of epigenetic (and more) treatments for cancer. Nat Rev Cancer 2006; 6: 38-51. https://doi.org/10.1038/ nrc1779

[9] REICHERT N, CHOUKRALLAH MA, MATTHIAS P. Multiple roles of class I HDACs in proliferation, differentiation, and development. Cell Mol Life Sci 2012; 69: 2173-2187. https://doi.org/10.1007/s00018-012-0921-9

[10] ELLIS L, ATADJA PW, JOHNSTONE RW. Epigenetics in cancer: targeting chromatin modifications. Mol Cancer Ther 2009; 8: 1409-1420. https://doi.org/10.1158/1535-7163. MCT-08-0860

[11] ELLIS L, PILI R. Histone Deacetylase Inhibitors: Advancing Therapeutic Strategies in Hematological and Solid Malignancies. Pharmaceuticals (Basel) 2010; 3: 2411-2469. https://doi.org/10.3390/ph3082441
[12] CHOUDHARY C, KUMAR C, GNAD F, NIELSEN ML, REHMAN $\mathrm{M}$ et al. Lysine acetylation targets protein complexes and co-regulates major cellular functions. Science 2009; 325: 834-840. https://doi.org/10.1126/science.1175371

[13] CHOU CW, WU MS, HUANG WC, CHEN CC. HDAC inhibition decreases the expression of EGFR in colorectal cancer cells. PLoS One 2011; 6: e18087. https://doi.org/10.1371/ journal.pone.0018087

[14] LABONTE MJ, WILSON PM, FAZZONE W, RUSSELL J, LOUIE SG et al. The dual EGFR/HER2 inhibitor lapatinib synergistically enhances the antitumor activity of the histone deacetylase inhibitor panobinostat in colorectal cancer models. Cancer Res 2011; 71: 3635-3648. https://doi. org/10.1158/0008-5472.CAN-10-2430

[15] FREW AJ, JOHNSTONE RW, BOLDEN JE. Enhancing the apoptotic and therapeutic effects of HDAC inhibitors. Cancer Lett 2009; 280: 125-133. https://doi.org/10.1016/j.canlet.2009.02.042

[16] SUN S, HAN Y, LIU J, FANG Y, TIAN Y et al. Trichostatin A targets the mitochondrial respiratory chain, increasing mitochondrial reactive oxygen species production to trigger apoptosis in human breast cancer cells. PLoS One 2014; 9: e91610. https://doi.org/10.1371/journal.pone.0091610

[17] MA J, GUO X, ZHANG S, LIU H, LU J et al. Trichostatin A, a histone deacetylase inhibitor, suppresses proliferation and promotes apoptosis of esophageal squamous cell lines. Mol Med Rep 2015; 11: 4525-4531. https://doi.org/10.3892/ mmr.2015.3268

[18] WATSON JA, MCKENNA DJ, MAXWELL P, DIAMOND J, ARTHUR K et al. Hyperacetylation in prostate cancer induces cell cycle aberrations, chromatin reorganization and altered gene expression profiles. J Cell Mol Med 2010; 14: 16681682. https://doi.org/10.1111/j.1582-4934.2009.00835.x

[19] YANG X, FERGUSON AT, NASS SJ, PHILLIPS DL, BUTASH KA et al. Transcriptional activation of estrogen receptor alpha in human breast cancer cells by histone deacetylase inhibition. Cancer Res 2000; 60: 6890-6894.

[20] DAMASKOS C, VALSAMI S, KONTOS M, SPARTALIS E, KALAMPOKAS T et al. Histone Deacetylase Inhibitors: An Attractive Therapeutic Strategy Against Breast Cancer. Anticancer Res 2017; 37: 35-46. https://doi.org/10.21873/anticanres. 11286

[21] CHEN L, JIN T, ZHU K, PIAO Y, QUAN T et al. PI3K/ mTOR dual inhibitor BEZ235 and histone deacetylase inhibitor Trichostatin A synergistically exert anti-tumor activity in breast cancer. Oncotarget 2017; 8: 11937-11949. https:// doi.org/10.18632/oncotarget.14442

[22] LAW ME, CORSINO PE, JAHN SC, DAVIS BJ, CHEN S et al. Glucocorticoids and histone deacetylase inhibitors cooperate to block the invasiveness of basal-like breast cancer cells through novel mechanisms. Oncogene 2013; 32: 13161329. https://doi.org/10.1038/onc.2012.138

[23] GERARD C, GOLDBETER A. From quiescence to proliferation: Cdk oscillations drive the mammalian cell cycle. Front Physiol 2012; 3: 413. https://doi.org/10.3389/ fphys.2012.00413 
[24] AGUERO MF, FACCHINETTI MM, SHELEG Z, SENDEROWICZ AM. Phenoxodiol, a novel isoflavone, induces G1 arrest by specific loss in cyclin-dependent kinase 2 activity by $\mathrm{p} 53$-independent induction of p21WAF1/CIP1. Cancer Res 2005; 65: 3364-3373. https://doi.org/10.1158/0008-5472. CAN-04-2429

[25] QUEIROZ AB, FOCCHI G, DOBO C, GOMES TS, RIBEIRO DA et al. Expression of p27, p21(WAF/Cip1), and p16(INK4a) in normal oral epithelium, oral squamous papilloma, and oral squamous cell carcinoma. Anticancer Res 2010; 30: 2799-2803.

[26] PARK H, LEE YJ, KIM TH, LEE J, YOON S et al. Effects of trichostatin A, a histone deacetylase inhibitor, on the regulation of apoptosis in H-ras-transformed breast epithelial cells. Int J Mol Med 2008; 22: 605-611.

[27] ZHANG J, ROBERTS TM, SHIVDASANI RA. Targeting PI3K signaling as a therapeutic approach for colorectal cancer. Gastroenterology 2011; 141: 50-61. https://doi. org/10.1053/j.gastro.2011.05.010

[28] CHEN CS, WENG SC, TSENG PH, LIN HP, CHEN CS. Histone acetylation-independent effect of histone deacetylase inhibitors on Akt through the reshuffling of protein phosphatase 1 complexes. J Biol Chem 2005; 280: 38879-38887. https://doi.org/10.1074/jbc.M505733200
[29] GARMPIS N, DAMASKOS C, GARMPI A, KALAMPOKAS E, KALAMPOKAS T et al. Histone Deacetylases as New Therapeutic Targets in Triple-negative Breast Cancer: Progress and Promises. Cancer Genomics Proteomics 2017; 14: 299-313. https://doi.org/10.21873/cgp.20041

[30] FEDELE P, ORLANDO L, CINIERI S. Targeting triple negative breast cancer with histone deacetylase inhibitors. Expert Opin Investig Drugs 2017; 26: 1199-1206. https://doi.org/10 $.1080 / 13543784.2017 .1386172$

[31] WERNER TL, RAY A, LAMB JG, VANBROCKLIN M, HUEFTLE K et al. A Phase I Study of Neoadjuvant Chemotherapy With Nab-Paclitaxel, Doxorubicin, and Cyclophosphamide in Patients With Stage II to III Breast Cancer. Clin Breast Cancer 2017; 17: 503-509. https://doi.org/10.1016/j. clbc.2017.04.010

[32] HSIEH HY, CHUANG HC, SHEN FH, DETROJA K, HSIN LW et al. Targeting breast cancer stem cells by novel HDAC3-selective inhibitors. Eur J Med Chem 2017; 140: 42-51. https://doi.org/10.1016/j.ejmech.2017.08.069

[33] WITT AE, LEE CW, LEE TI, AZZAM DJ, WANG B et al. Identification of a cancer stem cell-specific function for the histone deacetylases, HDAC1 and HDAC7, in breast and ovarian cancer. Oncogene 2017; 36: 1707-1720. https://doi. org/10.1038/onc.2016.337 